\title{
TARGET DETECTION USING MULTI RESOLUTION ANALYSIS FOR CAMOUFLAGED IMAGES
}

\author{
Chennamsetty Pulla Rao ${ }^{1}$, Dr. A Guruva Reddy ${ }^{2}$ and Dr. C.B. Rama Rao ${ }^{3}$ \\ ${ }^{1}$ Research scholar, Department of ECE, JNTU Kakinada \\ ${ }^{2}$ Professor, Department of ECE, MIC College of Technology \\ ${ }^{3}$ Associate professor, Department of ECE, NIT Warangal
}

\begin{abstract}
Target detection is a challenging problem having many applications in defense and civil. Most of the targets in defense are camouflaged. It is difficult for a system to detect camouflaged targets in an image. A novel and constructive approach is proposing to detect object in camouflage images. This method uses various methodologies such as 2-D DWT, gray level co-occurrence matrix (GLCM), wavelet coefficient features, region growing algorithm and canny edge detection. Target detection is achieved by calculating wavelet coefficient features from GLCM of transformed sub blocks of the image. Seed block is obtained by evaluating wavelet coefficient features. Finally the camouflage object is highlighted using image processing schemes. The proposed target detection system is implemented in Matlab 7.7.0 and tested on different kinds of images.
\end{abstract}

\section{KEYWORDS}

Canny edge detection, Region growing, seed block, Wavelet decomposition

\section{INTRODUCTION}

Target detection in camouflaged images is one of the most important applications of computer vision. Target detection in camouflaged images is an approach by which we identify one or group of target objects in scene. It is very easy for a human to identify different objects in image but it's difficult for a computer program to identify different objects [2]. In target detection in camouflaged images, images are of different types such as visual, Aerial, IR, etc and they are under different categories (stationary target, moving target) and environments (atmospheric turbulences). It is difficult for a computer program to detect target in these restrictions [3]. Till now so many researches are going on this approach. Some of those approaches [8] are like using pattern recognition, wavelets, texture, connectivity component based approach, descriptors based methods and traditional thresholding methods so on. In developing a system, there are many difficulties like recognition accuracy, occupation of size (image) and execution time so on. These are the things that motivated me to solve some of those issues. My concentration is towards the approach that based on wavelet decomposition and wavelet coefficient features.

DOI: $10.5121 /$ ijci.2016.5416 


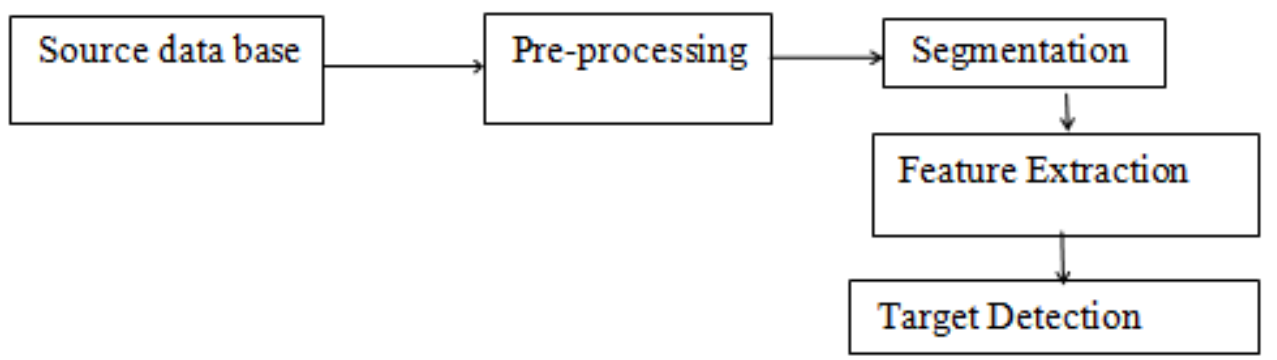

Figure 1. Simplified model for target detection system

The approach of this paper is to build a constructive approach for target detection in camouflaged images that recognize the targets in different kinds of images. To make a system that can able to take images of any size, colour or gray, and related to different environments. It should able to detect targets of any size and in any environment. Target detection in camouflaged images can be done using several approaches; a simplified model is shown in figure 1 . Wavelet transform is chosen as it is one of the novel techniques for solving target detection problem.

Camouflage is a natural phenomenon of many animals in the nature. It is an advantage to them, so that they can protect themselves from their enemies. The similar camouflage technique is used in military applications. Generally in military applications, camouflage paint patterns are used for concealing soldiers and their equipment from being detected by the enemy, making them appear to be part and parcel of the natural environmental conditions surrounding them. The extent to which we can camouflage will only be limited by our imagination. Different items are used for these purpose but the ones that standout are face painting, leaves also some fabrics are dyed in shades of green, tan, black, brown so as to make the wearer indistinguishable from the surroundings. Wearer may be a human being, or any other equipment.

Different camouflage paint patterns are used depending on the seasonal changes and the type of terrain used. The standard camouflage colors are blue, regular, desert and woodland. From these basic colors we can come up with desert sand, forest green, light green, white, earth yellow among other colors. Colors should always be chosen from the base color followed by the highlights. The surrounding areas will always determine the type of pattern and colors to use.

\section{SCOPE OF APPROACH}

Under different types of atmospheric turbulences and different kinds of targets under different environments with the help of seed blocks it is possible to recognize the objects in images. So all these approaches are characterize with the help of multi resolution analysis, similarly by the technique of canny edge detection invented by canny is using to extract the edges of target in the camouflaged kinds of images as explained above.

\section{SELECTED METHODS}

There are many methods provided by researchers for recognition of objects. In those few of methods are met their requirements. Based on the advanced computer vision approaches here we 
suggested the approach like using multi resolution analysis with traditional edge detection and seed block approaches.

Filter bank structure is the simple implementation of 1-D DWT, whereas image processing applications requires two dimensional implementation of wavelet transform [4]. Implementation of 2-D DWT is also referred to as ,multidimensionale wavelet transform. The multiresolution analysis as shown in Figure 3.1 This process continues until some final scale is reached.

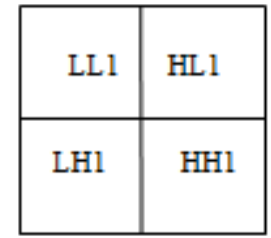

(a)

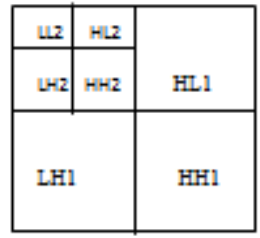

(b)

Figure 3.1: (a) One level wavelet transform (b) Two level wavelet transform

From the obtained gray level co-occurrence matrix, significant wavelet coefficient features (WCFs) preferably contrast, cluster shade, and cluster prominence are evaluated [1]. The features are selected for logarithmic normalization on their dynamic range of values. Several parameters associated with the co-occurrence matrix are contrast, energy, entropy, cluster prominence, cluster shade, and local homogeneity. As we know how the GLCM from the Harlick suggested method. From the obtained gray level co-occurrence matrix, significant wavelet coefficient features are computed using the formulae given below [2]

Coming to the edge detection technique the traditional technique is The Canny edge detection operator was developed by John F. Canny in 1986 this method works better compared to sobel and prewitt operators. The Canny edge detection algorithm is known to many as the optimal edge detector. Canny proposed some criteria to improve the earlier edge detection methods. An image and its canny detected image are shown in the following figures 3.3.

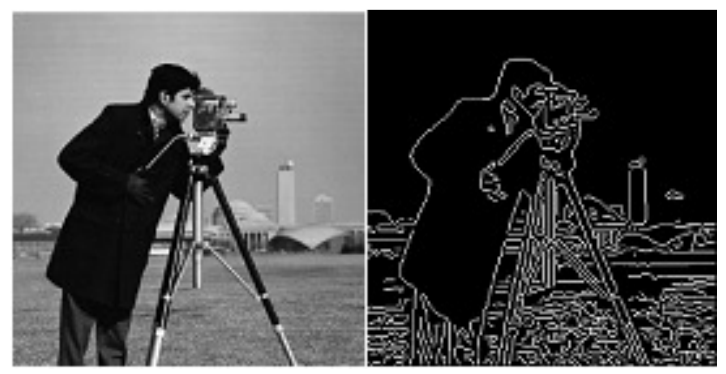

Figure 3.3: Original and Canny edge detected image 


\section{Proposed Method}

Identification of object in camouflaged images is a difficult approach due to the stochastic nature of background from the foreground. So traditional approaches and processing schemes is will not provide a satisfactory results due to its randomness in order to solve the problem in camouflage images object identification proposing a constructive approaches witch will overcome the problems in traditional processing schemes. In the proposing approach the results are found to be satisfactory. The block diagram for the proposed Target Detection in Camouflaged images system $[1,2]$ is given in the figure4.1 Input image. Similarly the Process flowchart shows all the steps involved in this proposed method is shown in figure $\mathbf{4 . 2}$

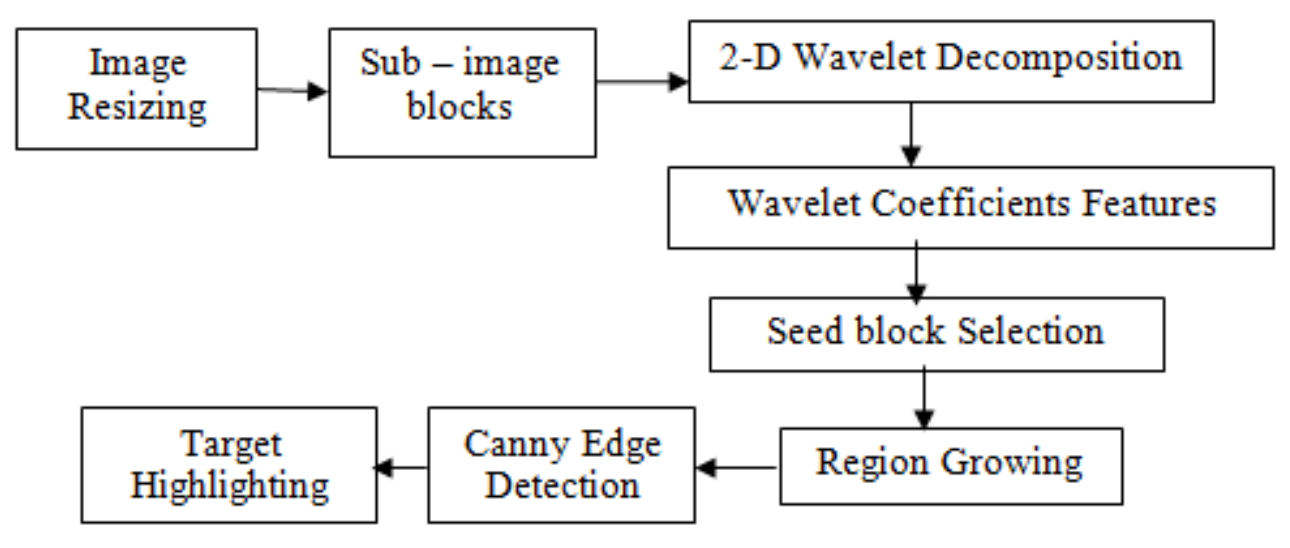

Figure 4.1: Target detection system 


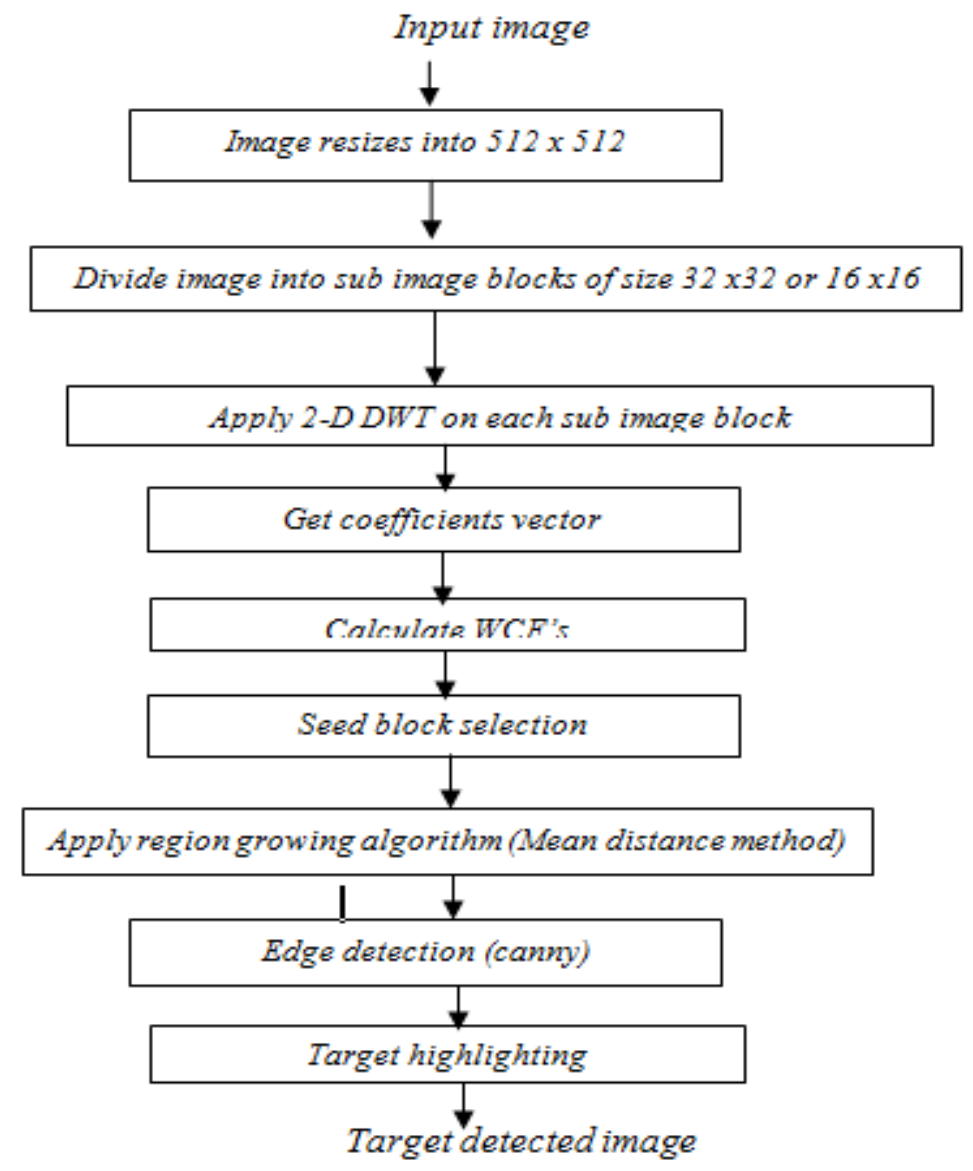

Figure 4.2: Flow chart of proposed target detection system

\section{A. Image resizing:}

Consider a camouflage image of different dimensions such that we can divide it into $32 \times 32$ or 16 $\mathrm{x} 16$ in the next step. Generally in this system we resizes into 512 x512. If the assumed image is color image convert it in to gray scale image for better processing. Then in the further steps we can process easily and able to calculate GLCM [7]. It is shown in the figure 4.3.

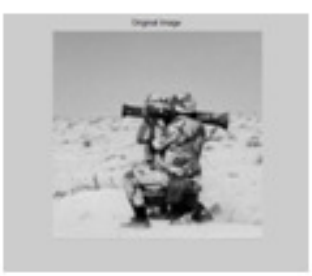

(a)

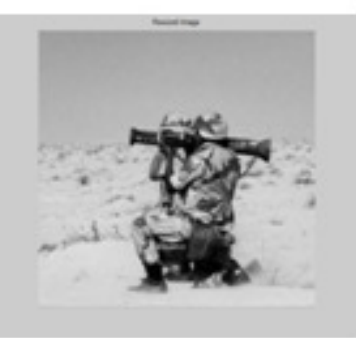

(b)

Figure 4.3: (a) Original image of size 356 × 355 x 3, (b) Resized image of size $512 \times 512$ 


\section{B. Making sub image blocks:}

This proposed system works on the principle of fixed window size technique. If image is resized into $512 \times 512$, and if we divide into sub images of each size $32 \times 32$, we get 256 sub images. If we divide into $16 \times 16$, we get 512 sub images. Block size depends up on the dimension of the object and the image.

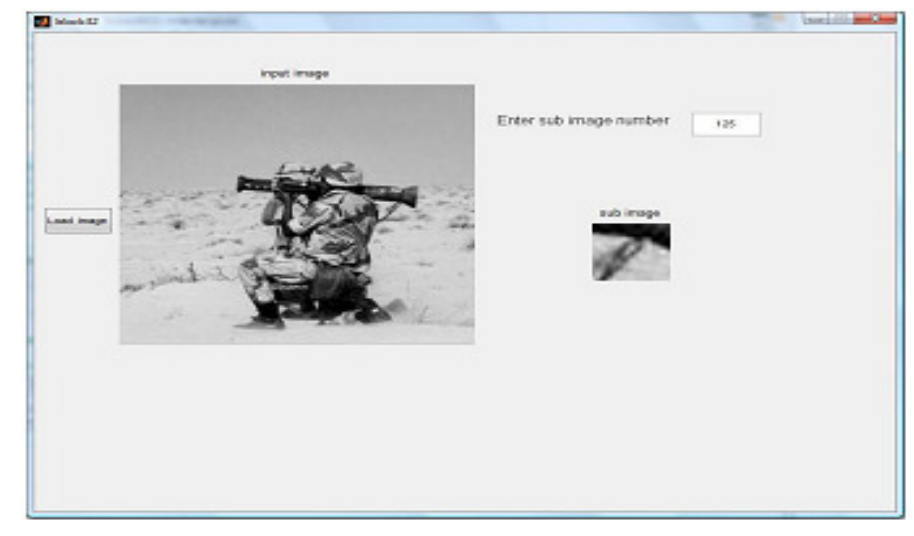

Figure 4.4: Sub image block

Once sub images are obtained, we have to process each sub image separately in the further steps. Finally we have to select seed block i.e., sub image block having the highest sum of wavelet coefficient features. Using the seed block, processing techniques are used to detect the target

\section{DWT Decomposition:}

After dividing input image into sub image blocks, each sub image block is decomposed using 2D DWT. Different wavelets are there to apply such as Haar, Daubechies, Meyer, Morlett and Symlets. In the proposed method Daubechies wavelet is used which is of order 2. It is represented as db2. If the size of sub block is more the level of decomposition is to be large in number if it is not so then small number level of decomposition is enough for multi resolution analysis purpose. Level of decomposition is 2 for this system. After decomposition, we get wavelet coefficients. The lower level in decomposition gives coarse level of coefficients which means approximation coefficients and higher levels give finest scale wavelet coefficients which means detail coefficients.

If image is resized into 512 × 512 and divided into sub images of size $32 \times 32,256$ sub images are obtained. Then after applying DWT decomposition 256 wavelet coefficients vectors are obtained.

\section{Feature extraction:}

From the transformed sub block of camouflage image features are evaluated by statistical means to find the seed block details from set of sub blocks to process further. The feature are calculating 
by using gray level co-occurrence matrices which is a statistical model based on the probability of occurrence of gray tone values. The statistical features based on GLCM which are cluster prominence, cluster shade and contrast used to evaluate seed block from a set of sub block. The seed block is identified by highest sum of statistical features in each sub block.

\section{Region growing process:}

Region growing is a region based segmentation process in which sub regions are grown into larger regions based on predefined criteria such as threshold and adjacency. In developed approach, the region growing algorithm is based on mean distance method [1].

In this method, the first step is to sort the feature values of all the windows, that is, sub image blocks in ascending order so that the window whose value is the largest would be the seed window. The threshold is determined by finding the average (A) of the first $n \%$ of the windows, which are adaptively chosen depending upon the target image. Now, the statistical feature values like (cluster details and contrast) of all the 8-adjacent blocks are compared with the average value, $A$, and the $S_{\text {high }}$ value. The window whose value is closer to $S_{\text {high }}$ (obtained from the seed block) value will be merged with the seed window. This process is repeated for all 8 adjacent blocks. If no window is merged from the 8 adjacent blocks, then the algorithm terminates. If at least one window is merged from the 8 adjacent blocks, then the above procedure will be repeated with the 16 adjacencies and so on.

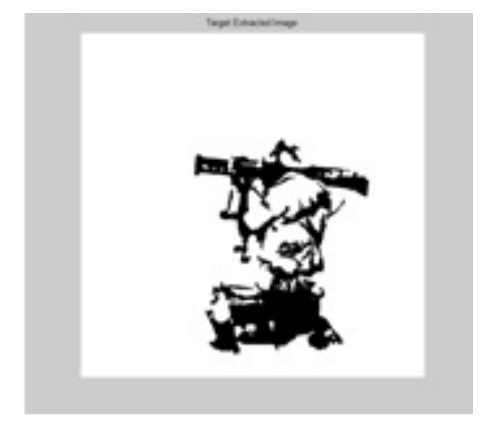

Figure 4.5: Image after applying region growing process.

\section{E. Edge detection and Target highlighting}

Canny edge detector was one of the good edge detection technique to extract edge information. Now we apply canny edge detection algorithm to find the edges in the region grown image. It will give all the edges present in the region grown image. It gives edges of the target in the scene. This step is used to highlighting step. Image after applying canny edge detection is shown in the figure 4.6 


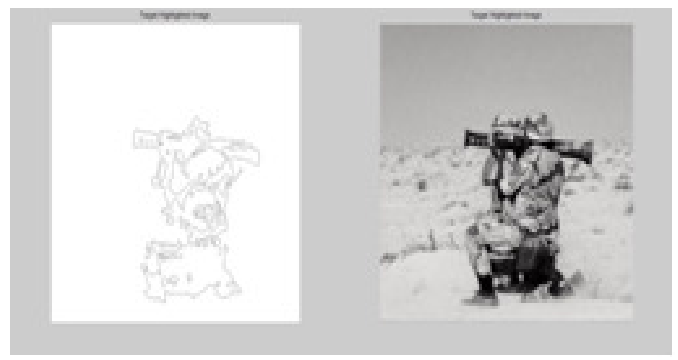

(a)

(b)

Figure 4.6: (a) Canny edge detected image (b) Target highlighted image

Canny edge detected image is presently in gray level type. This is converted to indexed image and then converted to RGB image. Then it is added to the original image to highlight the target in the scene.

\section{SiMULATION RESULTS}

The simulation of the proposed Target Detection in Camouflaged images system is simulated on Matlab 7.7.0 (R2008b). The proposed approach is tested on different kind of images, which are of different sizes and in different environments. The input images may be colour or gray scale and are of any resolution. These images contain different type of targets such as camouflaged soldiers in war environment, camouflaged tankers, aero planes, birds and statues

\section{Camouflaged soldiers in war environment}

2. Camouflaged soldier in green fields

The simulation result for image 1 is discussed in the previous section. Now the simulation result for image 2 is explained here. For every input image we have resized image, target extracted image, Canny edge detected image, target highlighted image. Sizes of original image, resized image, seed block, seed point (x, y) are calculated. Profiles of wavelet coefficient features such as cluster features and contrast are shown. Details of the seed block (sub image block) are given

\section{Camouflaged soldiers in green fields}

This is an image contains a soldier camouflaged (disguised) in green fields. For this wavelet coefficient features are calculated and then seed block is found. Details of the seed block are given and its sub image block is shown.

Size of the original image $=180 \times 150 \times 3$

Size of the resized image $=512 \times 512$ 


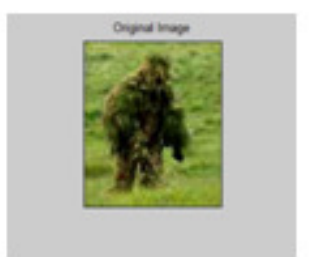

(a)

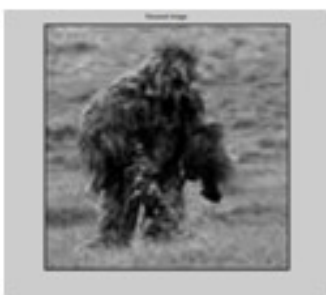

(b)

Figure 5.1: (a) Original image of size 180 x 150 x 3(b) Resized image of size 512 x512

\section{A. Wavelet coefficient features}

After applying wavelet decomposition to all sub image blocks, we get coefficients vector. Gray level co-occurrence matrix is calculated from transformed coefficients. Then statistical features are evaluated for further processing techniques

Now WCFees are calculated for all the sub image blocks and they are plotted as above. Contrast is normalized linearly and the other two are normalized logarithmically. Sub image with maximum of the sum of the features is treated as a seed block. Seed point is the centre point of the seed block. Seed point is used in the region growing algorithm. Now $\mathrm{WCF}^{\mathrm{ec}} \mathrm{s}$ are calculated for all the sub image blocks and they are plotted as above. Contrast is normalized linearly and the other two are normalized logarithmically. Sub image with maximum of the sum of the features is treated as a seed block. Seed point is the centre point of the seed block.
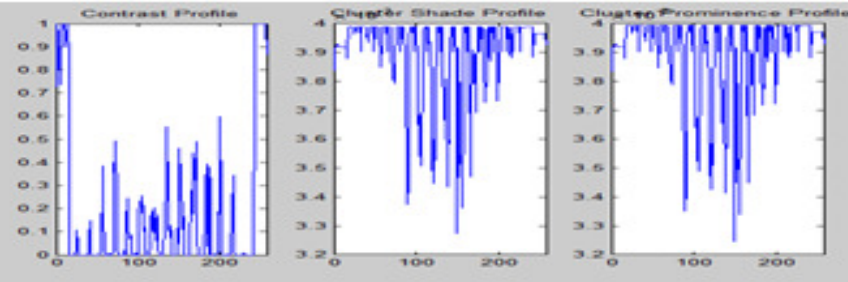

Figure 5.2: Profiles of wavelet coefficient features

\section{B. Seed block details:}

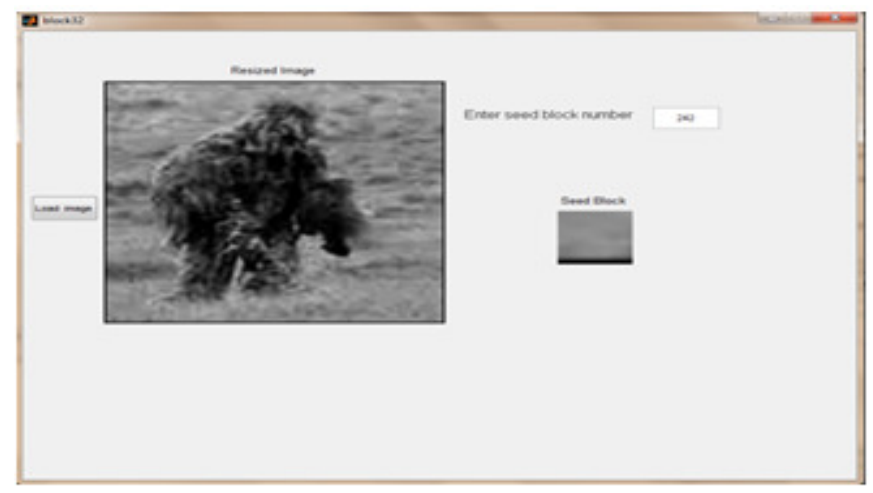

Figure 5.3: GUI of Seed block 
Seed block $=242$ th sub image block

Seed point on the resized image $=(497,49)$

Wavelet coefficient features for the seed block shown above:

Contrast $=490$

Cluster_shade $=2.2379 \mathrm{e}+011$

Cluster_prominence $=3.0319 \mathrm{e}+014$

\section{Region grown, canny edge detected, and target highlighted image:}

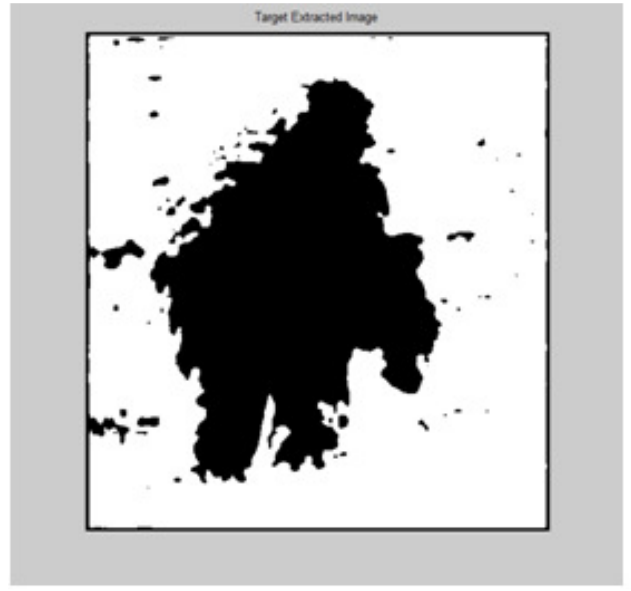

Figure 5.4: Region grown image

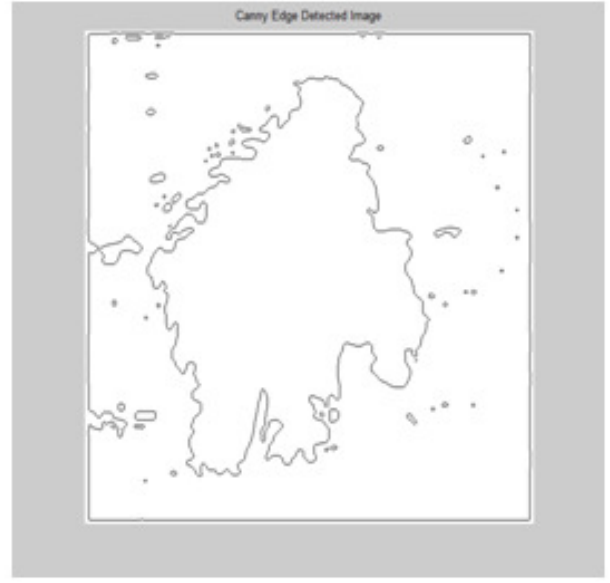

Figure 5.5: Canny edge detected image

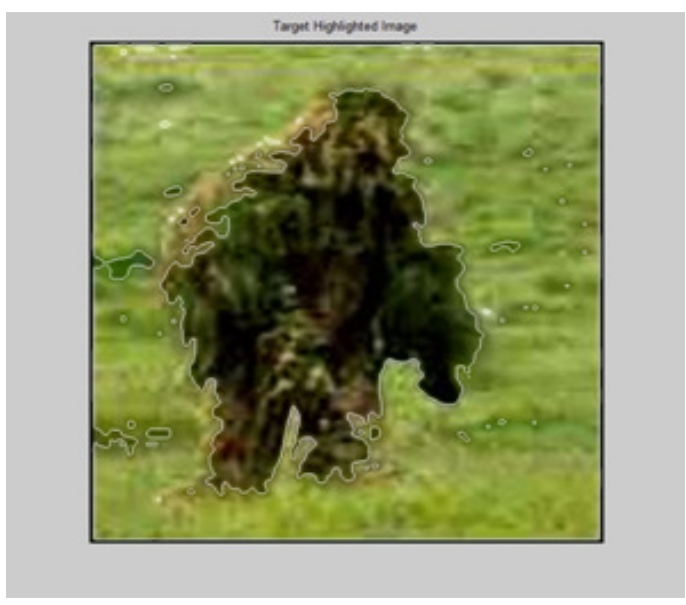

Figure 5.6: Target highlighted image

\section{Tabulations:}

Proposed Target Detection in Camouflaged images system is implemented on Matlab7.7.0 (R2008b) and tested on these images. All these information is calculated in the above shown simulation. Seed block number, seed point and wavelet coefficient features for different images are tabulated as shown in the table given below: 
International Journal on Cybernetics \& Informatics (IJCI) Vol. 5, No. 4, August 2016

\begin{tabular}{|l|l|l|l|l|l|l|}
\hline S. No & \multicolumn{1}{|c|}{ Image } & $\begin{array}{l}\text { Seed } \\
\text { Block }\end{array}$ & $\begin{array}{c}\text { Seed } \\
\text { Point }\end{array}$ & Contrast & Cluster shade & $\begin{array}{l}\text { Cluster } \\
\text { promin- ence }\end{array}$ \\
\hline $\mathbf{1}$ & $\begin{array}{l}\text { Camouflaged } \\
\text { soldiers in war } \\
\text { environment }\end{array}$ & 107 & $(209,337)$ & 750 & $1.2827 \mathrm{e}+011$ & $1.4436 \mathrm{e}+014$ \\
\hline $\mathbf{2}$ & $\begin{array}{l}\text { Camouflaged } \\
\text { soldier in } \\
\text { green fields }\end{array}$ & 242 & $(497,49)$ & 490 & $2.2379 \mathrm{e}+011$ & $3.0319 \mathrm{e}+014$ \\
\hline
\end{tabular}

The proposed method is tested on different kinds of images in different environments

\begin{tabular}{|l|l|l|l|l|l|l|}
\hline $\mathbf{1}$ & Aero plane & 103 & $(209,209)$ & 47 & $1.9077 \mathrm{e}+011$ & $2.4506 \mathrm{e}+014$ \\
\hline $\mathbf{2}$ & Bird & 106 & $(209,305)$ & 202 & $2.5880 \mathrm{e}+011$ & $3.6802 \mathrm{e}+014$ \\
\hline $\mathbf{3}$ & Statue & 170 & $(337,305)$ & 77 & $1.6634 \mathrm{e}+011$ & $2.0414 \mathrm{e}+014$ \\
\hline $\mathbf{4}$ & Ship & 217 & $(433,273)$ & 446 & $9.4556 \mathrm{e}+010$ & $9.6129 \mathrm{e}+013$ \\
\hline
\end{tabular}

\section{CONCLUSIONS AND FUTURE SCOPE}

The proposed techniques for detecting targets in camouflaged images and is tested on different kinds of images. Wavelet based approach is adapted to encounter the multiresolution analysis problem in images like camouflaged images due to randomness in scene. Statistical features are used as performance metrics in this implementation. These metrics are very useful in the object detection for camouflaged images. Canny edge operator is appropriate to detect the edges of the extracted image. The proposed system is implemented on Matlab 7.7.0 (R2008b). Performance of the algorithm is tested on images of arbitrary sizes, gray or colour, and related to different environments and the results are convincing. In future, the proposed method can be extended further at different steps. After resizing the original image, we can divide into sub image blocks of different dimensions according to the size of the object. Based on the features of the obtained target we can classify it. It can be extended to detect multiple targets in an image. Performance of the algorithm can be assessed by changing the wavelets and level of decomposition.

\section{ACKNOWLEDGEMENT}

As part of research work it was happy to express sincere thanks to supervisor Dr A Guruva Reddy of his continuous suggestions and help and also to Co-Supervisor Dr C.B. Rama Rao on completeness of this research work in a stipulated time with his monitoring and suggestions. 
International Journal on Cybernetics \& Informatics (IJCI) Vol. 5, No. 4, August 2016

\section{REFERENCES}

[1] Sujit K Singh, Chitra A Dhawale and Sanjay Misra, "Survey of Object Detection Methods in Camouflaged Image", Proc: IERI, PP. 1-6, ELSEVIER, 2012.

[2] Yuxin Pan, Yiwag Chen, Qiang Fu, Ping Zhang \& Xin Xu, "Study on the Camouflaged target Detection Method Based on 3D Convexity", Proc: Modern Applied Science , Vol. 5, PP. 152-157, 2011.

[3] Dileep Kumar, Dr. Shrishu Varma, "Multiresolution framework with Neural Network approach for Automatic Target Recognition (ATR)", Proc: ICSAAP, PP. 168-173, IEEE computer society, 2009.

[4] B. Ravichandran, Avinash Gandhe, Robert Smith, Raman Mehra, "Robust automatic target recognition using learning classifier systems", Proc: INFORMATION FUSION, PP. 252-265, ELSEVIER ScienceDirect 2007.

[5] Joao Paulo Pordeus Gomes, Jose Fernando Basso Brancalion, and david Fernandes, "Automatic target Recognition in Synthetic Aperture Radar Image Using Multiresolution Analysis and Classifiers Combination", IEEE, 2008.

[6] Hung-Kuo Chu, Wei-Hsin Hsu, Niloy J. Mitra, Daniel Cohen-Or, Tien-Tsin Wong, Tong-Yee Lee, "Camouflage Images", ACM transaction on Graphic, 2010.

[7] S. Arivazhagan, L. Ganesan, V. Angayarkanni, "Color Texture Classification using Wavelet Transform", Proc: ICCIMA05,IEEE, 2005.

[8] S. Z. Mahmoodabadi, A. Ahmadian, M. D. Abolhasani, M. Eslami, J. H. Bidgoli, "Feature Extraction Based on Multiresolution Wavelet Transform", IEEE proceedings , PP. 3902-3905, 2005.

[9] J. Manikandan, B. Venkataramani and M. Jayachandran, "Evaluation of Edge Detection Techniques towards Implementation of automatic target Recognition", Proc: Computational Intelligence and Multimedia applications, PP. 441-445, 2007.

[10] Zeyong Shan and Selin Aviyente, "Image Denoising Based on the Wavelet Co-Occurrence matrix", Proc:ICASSP2005 , PP. 645-648, IEEE 2005.

[11] Du-Ming Tsai, Cheng-Huei chiang, "Rotation -invariant pattern matching using wavelet decomposition", Proc: Pettern recognition Letters, PP. 191-201, ELSEVIER Pattern recognition Letters 2002.

[12] G. Van de Wouwer, P. Scheuders and D. Van Dyck, "Statistical Texture Characterization from Discrete Wavelet Representations" Proc: IEEE TRANSACTIONS ON IMAGE PROCESSING , Vol.8, PP. 592-598, 1999.

[13] Robert M. Haralick, K. Shanmugam and Itshak Dinstein, "Textual Features for Image Classification", Proc: IEEE TRANSACTIONS ON SYSTEMS, MAN, AND CYBERNETICS, vol SMc-3,NO.6,,PP. 610-621, 2010.

[14] Ch. S. Sastry, Arun K. Pujari, B. L. Deekshatulu, C. Bhagvati, "A Wavelet based Multiresolution algorithm for rotation invariant feature extraction", Proc: Pattern Recognition Letters, vol. 25, PP. 1845-1855, ELSEVIER Pattern Recognition Letters 2004.

[15] Sirui Tian, Chao Wang, Hong Zhang, Bo Zhang, Fan Wu, "A Wavelet Based Targets Detection Method for high resolution Airborne SAR Data ", Proc: IEEE transactions of Geosciences and Remote Sensing Symposium , PP. 2071-2073, 2007.

[16] Bir Bhanu, Automatic Target Recognition: State of the Art Survey, IEEE Transactions on Aerospace and Electronic Systems, Vol. AES-22, No. 4, July 1986

[17] Rafael C. Gonzalez and Richard E. Woods, "Digital image processing", Pearson education, Inc, 1999.

[18] E. Hosseini, Aria M.R. Saradjian, J. Amini and C. Lucas, "Generalized co-occurrence Matrix to Classify IRS-1D Images using Neural Network", 20th ISPRS Congress, Istanbul, Turkey, July 2004.

[19] Michael W. Roth, "Survey of Neural Network Technology fot Automatic Target Recognition", IEEE Transactions on Neural Networks, Vol. 1. 
International Journal on Cybernetics \& Informatics (IJCI) Vol. 5, No. 4, August 2016

\section{AUTHORS}

Chennamsetty Pulla Rao received his Bachelors of Degree in Electronics and Telecommunication Engineering from IETE in 2007. Received Master's degree in Advanced Communication Systems from National Institute of Technology, Warangal in 2010and pursuing P h D at JNTU Kakinada. At present working as Associate Professor in MIC College of technology from July 2010.Published one National and one International Journal research interests are image processing techniques and Wireless Communications.

Dr. A Guruva Reddy working as Professor in Electronics \& communication engineering at DVR \& Dr H.S MIC college of Technology in INDIA. Completed Doctorial degree from JNTU Ananthapur in the year of 2010. He has 15 international and national journals in the area of image processing. Also organized and attended national level workshops like Funded by SERB and Faculty Development programmes. At present supervising five research scholars in the area of signal and image processing at JNTU Kakinada.

Dr. RAMA RAO C B working as Associate Professor in Electronics \& communication engineering at NIT Warangal in INDIA. Received Doctorial degree from IIT kharagpur in the year of 1995.Published 12 international and national journals in the area of signal and image processing. Also organized and attended several national level workshops like ISTE and STTP. At present supervising three research
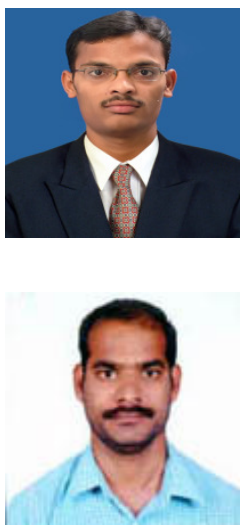
scholars in the area of signal and image processing at NIT Warangal. 